\title{
Influence of Citrus Source and Test Genotypes on Inoculations with Candidatus Liberibacter asiaticus
}

\author{
Ed Stover ${ }^{1}$, David G. Hall, and Robert G. Shatters, Jr. \\ U.S. Horticultural Research Laboratory, U.S. Department of Agriculture, \\ Agricultural Research Service, 2001 South Rock Road, Fort Pierce, FL \\ 34945
}

\section{Gloria A. Moore \\ Horticultural Sciences Department, University of Florida, 1111 Fifield Hall, Box 110690, Gainesville, FL 32611-0690}

Additional index words. Asian citrus psyllid, citrus greening, Diaphorina citri, disease resistance, disease tolerance, HLB, huanglongbing

Abstract. Assessments of the resistance of citrus germplasm to huanglongbing (HLB) can be expedited by inoculating plants under laboratory or greenhouse settings with the HLB bacterium, Candidatus Liberibacter asiaticus (CLas). Consistent rapid screening is critical to efficiently assess disease resistance among plant materials; however, a number of factors may govern the efficacy of such inoculations. Despite the rapidity at which HLB can spread in a grove, it often takes 8 to 10 months for high levels of CLas and HLB symptoms to develop even in highly susceptible sweet orange. Therefore, two experiments were conducted to assess factors that might influence efficiency in screening for HLB resistance. In one experiment, three test citrus genotypes ('Kuharske', previously shown to be HLB resistant; rough lemon, previously shown to be HLB tolerant; and 'Valencia', HLB susceptible) were bud grafted using CLas-infected buds from four different source genotypes. All bud source genotypes had similar levels of CLas titer, but citron, rough lemon, and Volkamer lemon were hypothesized to be better bud inoculum sources as they are more tolerant of HLB than 'Valencia'. Among the three test genotypes over all sources of infected buds, inoculations of 'Kuharske' resulted in lower CLas titers and fewer HLB symptoms than inoculations of rough lemon or 'Valencia'. Inoculations of rough lemon resulted in higher CLas titers and more pronounced HLB symptoms when it was inoculated using infected buds from rough lemon or 'Valencia'. Grafting 'Valencia' with infected buds from Volkamer lemon resulted in less disease than when 'Valencia' was grafted with infected citron, rough lemon, or 'Valencia' buds. Overall, these results suggest that the source of CLas-infected buds used to graftinoculate some genotypes will influence disease development. Trunk cross-sectional area increase for the year following infection was $3 \times$ higher in 'Kuharske' and rough lemon, compared with 'Valencia'. 'Kuharske' had very low levels of CLas (30 CLas/ $\mu \mathrm{g}$ DNA), whereas 'Valencia' (43,000 CLas/ $\mu \mathrm{g}$ DNA) and rough lemon (6700 CLas/ $\mu \mathrm{g}$ DNA) had relatively high levels. As an alternative to graft-inoculating plants with CLas-infected buds, plants can be subjected to infestations of CLas-infected Asian citrus psyllid (ACP) as occurs naturally. Of interest is if transmission rates of CLas and the development of HLB in a genotype are greater when the ACP have been feeding on the same host genotype. An experiment was therefore conducted to assess transmission of CLas by ACP reared on CLas-infected rough lemon to five different genotypes ('Carrizo', 'Flame' grapefruit, rough lemon, 'Temple', and 'Valencia'). These assessments were made using a detached leaf assay recognized as a faster method of gauging transmission rates of CLas than using whole plants. Higher percentages of ACP died when they were transferred from infected rough lemon to healthy 'Carrizo', and lower percentages died when they were transferred to rough lemon or 'Flame'. However, CLas transmission by infected ACP occurred to at least some leaves of each genotype in each of the five different assays, with $70 \%$ or more leaves of each genotype becoming infected in at least one assay. Over all assays, there was relatively little variation among genotypes in the percentage of leaves becoming CLas infected and in the titer of CLas developing in infected leaves. However, there were relatively large differences in transmission rates among individual assays unrelated to differences among test genotypes. Because of the rapidity of the detached leaf assay, efforts are merited to improve consistency of this inoculation method.

Huanglongbing disease is considered the greatest threat to the U.S. citrus industry (Gottwald, 2010). In the United States, HLB is associated with CLas and its insect vector, Diaphorina citri, the ACP. Following initial infection, symptoms may take many months to become evident. As the disease progresses, trees become less vigorous, with thinning canopies and abnormal leaves contributing to yield loss and poor fruit quality (Bové, 2006; Gottwald, 2010). Since HLB was first confirmed in Florida in 2005, it has been found in all 32 citrus producing counties in the state as well as in dooryard citrus in noncitrus producing counties (Halbert et al., 2008). It is becoming increasingly widespread in Texas, and several HLB-affected dooryard trees have now been identified in California (CDFA, 2015).

Management of HLB in Florida focuses on insecticide applications for vector control in combination with a series of production practices intended to reduce stress in infected trees. These practices have markedly increased production costs per hectare, while productivity in infected Florida orchards is reported to show an estimated average reduction of $41 \%$ compared with healthy orchards (Singerman and Useche, 2015). A more sustainable approach for the management of HLB would be the cultivation of citrus cultivars which are unaffected, or less affected, by the disease rather than more susceptible types (Gottwald, 2010).

Several studies indicate differences in HLB susceptibility among citrus cultivars and/or species, which may be sufficient to be economically significant (Stover and McCollum, 2011; Stover et al., 2015a, 2016). However, despite the rapid and devastating spread of HLB throughout Florida, it is remarkably difficult to quickly and efficiently screen for relative resistance and tolerance to HLB. Following graft-inoculation or exposure of germplasm to CLas-infected ACP, it often takes 8-10 months to observe a high level of CLas infection even in highly susceptible sweet orange. In discussions with many scientists studying HLB, several anecdotal observations suggested opportunities to improve inoculation efficiency.

Many graft inoculations are made using buds from highly HLB-susceptible sweet orange which may have compromised health and reduced ability to transfer CLas. Therefore, this study investigates whether a graftinoculum source showing more tolerance to HLB would enhance CLas transfer and facilitate identifying differences in HLB susceptibility

Some researchers reported that transition of ACP from one genotype to another appeared to compromise ACP health and consequent transfer of CLas. Therefore, we also examined whether ACP, reared on a specific test genotype and infected with CLas, could transfer CLas to the same test genotype at a higher rate than to other genotypes. This was evaluated using a detached leaf inoculativity test that was reported to be a faster approach to assessing CLas transmission rates than using whole plants (Ammar et al., 2013).

\section{Materials and Methods}

All experimental trees were maintained in a greenhouse at the U.S. Horticultural 
Table 1. Ten trees of each of three citrus genotypes ('Kuharske' citrange, rough lemon, and 'Valencia') were double bud inoculated 20 Mar. 2012 with highly HLB symptomatic citron, rough lemon, 'Valencia', or Volkamer lemon. In May 2012, trees were rebudded with the same source genotype if both prior buds were dead. For each bud, the leaf petiole was removed and assessed for CLas titer. Development of CLas titer was followed over time in inoculated trees and development of distinctive HLB mottling recorded. Petioles and leaf midribs from mature leaves, from flushes with some lignification on stems, were assessed for CLas titer using qPCR.

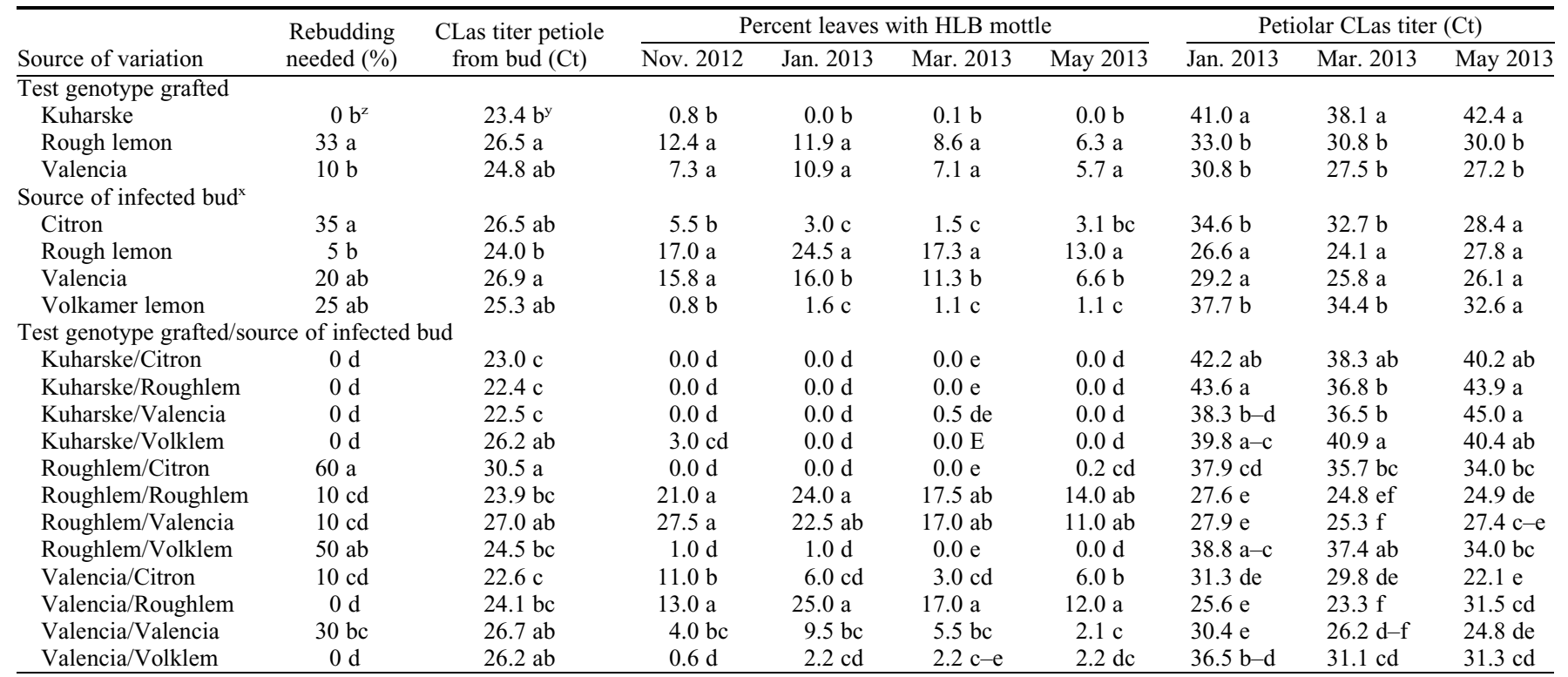

CLas = Candidatus Liberibacter asiaticus; $\mathrm{Ct}=$ cycle threshold; $\mathrm{HLB}=$ huanglongbing; $\mathrm{qPCR}=$ quantitative polymerase chain reaction.

${ }^{z}$ Within a column and section, means followed by the same letter are not significantly different at $P \leq 0.05$.

${ }^{\mathrm{y}} \mathrm{Ct}$ corresponds to genomes of CLas/ $\mu \mathrm{g}$ DNA as follows: $22=1,800,000,27=76,000,32=3300,37=140$, and $42=6$.

'Inoculation source data exclude the 'Kuharske' test trees since response was negligible in this genotype.

Research Laboratory (USHRL) in Fort Pierce, FL.

\section{Graft inoculations}

Six trees, each of 'Valencia' (Citrus sinensis, budded onto US-812, Citrus reticulata $\times$ Poncirus trifoliata), rough lemon (Citrus jambhiri, nucellar seedlings), citron (Citrus medica, cultivar Mhac Nao from rooted cuttings), and Volkamer lemon (Citrus volkamariana, nucellar seedlings) were graft inoculated on 20 Oct. 2010 with CLas using infected 'Valencia' budwood at three buds per tree, and these trees are hereafter referred to as source trees, because they were then used as sources of budwood for further graft inoculations. Rough lemon was shown to be more tolerant of HLB than 'Valencia', with sustained phloem transport even in heavily symptomatic leaves (Fan et al., 2012). Our observations indicate that Volkamer lemon and citron are also more HLB tolerant than sweet orange, and exceed rough

Received for publication 29 Feb. 2016. Accepted for publication 16 May 2016.

This project was funded in part through USDA/ APHIS. USDA is an equal opportunity provider and employer. Mention of trade names or commercial products in this article is solely for the purpose of providing specific information and does not imply recommendation or endorsement by the U.S. Department of Agriculture.

We thank Regina Tracy, Belkis Diego, Ashley Witkowski, Kathy Moulton, and Diane Helseth for their technical assistance in these projects.

${ }^{1}$ Corresponding author. E-mail: ed.stover@ars. usda.gov. lemon in maintaining relatively full canopies in HLB-infected trees, whereas 'Valencia' has substantial leaf loss and develops very thin canopies (Miles et al., 2016). The original inoculum of CLas was from an infected 'Lisbon' lemon (Citrus limon) from a commercial grove near Fort Pierce, FL (Albrecht and Bowman, 2008); it has been used extensively in experiments, with infected trees sustained through serial graft transmission.

These source trees developed distinctive HLB mottling and CLas titer, and provided material for graft inoculations of three test tree genotypes: 'Valencia' (budded on rough lemon), 'Kuharske' (C. sinensis $\times P$. trifoliata, from apomictic seed), and rough lemon (from apomictic seed). On 20 Mar. 2012, the three test genotypes were graft inoculated using buds from each of the four source genotypes. Two buds from the same source genotype were grafted onto each of 10 individuals of each test genotype, resulting in 10 trees each of 12 combinations (4 source genotypes $\times 3$ target genotypes). Source buds with attached leaves were randomly assigned to test trees. Buds were cut from branches clearly showing blotchy mottle symptoms, which is a distinctive symptom of HLB, and buds with attached leaves were randomly assigned to test trees. Two source buds of the same genotype were used for each test tree. The petioles and midribs of leaves from each bud were frozen for quantitative polymerase chain reaction (qPCR) of CLas titer with complete accounting of which leaf corresponded to which bud. On 31 May 2012, any tree where both inoculum buds were dead received an additional two buds from the same initial inoculum source. At the time of bud-inoculation, test trees had a diameter of 4 to $8 \mathrm{~mm}$ and a height of 0.5 to $0.9 \mathrm{~m}$. All trunk diameter measurements were made at $5 \mathrm{~cm}$ above the soil surface, which was just above the graft union on the budded trees.

After budding, data were collected seven times between June 2012 and June 2013 on trunk diameter and percentage of leaves showing distinct blotchy mottle symptoms on each test tree. Mature leaves from flushes with some lignification on stems were collected on the same dates and were assessed for CLas titer using qPCR.

\section{Detached leaf assays with ACP}

The experiments were conducted using the detached leaf assay described in Ammar et al. (2013). All ACP were adults from a colony maintained in a USHRL insectary on rough lemon which had been graft infected with the same CLas 'Lisbon' lemon source described above. CLas titer was determined on a subsample of colony ACP on the same date the leaves were exposed, using the method described in Hall et al. (2016). A single leaf was considered to be an experimental unit and ACP transfer was made to all leaves on the same day. Ten ACP were placed on 10 mature leaves each from 'Flame' grapefruit (Citrus paradisi), 'Temple' tangor $(C$. reticulata $\times C$. sinensis $)$, 'Valencia' sweet orange, 'Carrizo' ( $C$. sinensis $\times P$. trifoliata), and rough lemon. The experiment was conducted five times from Mar. 2013 to Feb. 2014, using leaf samples from trees with no recent insecticide 
applications. Sample leaves were checked to verify that no ACP mortality occurred within $24 \mathrm{~h}$ following placement. ACP mortality on the experiment leaves was also assessed at the end of the 1-week experimental feeding period. The leaves were incubated for an additional week and then CLas titer was determined based on qPCR of the petiole/ midrib of each leaf.

\section{Determination of CLas titer in leaves}

All leaf samples were stored at $-20{ }^{\circ} \mathrm{C}$ until processed. For each tree, the lower $2 \mathrm{~cm}$ from the leaf petiole base into the midrib were macerated in a Fast Prep 24 (MP Biomedicals, Santa Ana, CA) with DNA extracted from $200 \mathrm{mg}$ of macerated tissue using CTAB extraction followed by column clean up and concentration (Genesee Scientific, San Diego, CA). Each sample was assayed for CLas and citrus dehydrin via qPCR using an ABI 7500 thermocycler (Applied Biosystems, Foster City, CA). Each $25 \mu \mathrm{L}$ qPCR reaction was conducted using $100 \mathrm{ng}$ of template DNA (standardized using Nanodrop, Thermo Fisher Scientific, Waltham, MA) with primers specific to $16 \mathrm{~S}$ rDNA for CLas detection (Las Long): USHRL-CL1-L 5' -CTTACCAGCCCTTGACATGTATAGG-3' and USHRL-CL1-R 5'-TCCCTATAAAGTACCCAACATCTAGGTAAA-3' or Citrus dehydrin (CD-L 5'-TGAGTACGAGCCGAGTGTTG-3' and CD-R 5'-AAAACTTCACCGATCCACCAG- $3^{\prime}$ ). Citrus dehydrin was used as reference control to verify DNA quality and PCR amplification. The master mix consisted of Promega (Madison, WI) GoTaq qPCR with BRYT Green and $0.2 \mu \mathrm{M}$ of each primer using cycle parameters of $2 \mathrm{~min}$ at $95^{\circ} \mathrm{C}$ start, 50 cycles of $15 \mathrm{~s}$ at $95^{\circ} \mathrm{C}, 20 \mathrm{~s}$ at $60^{\circ} \mathrm{C}$, and $20 \mathrm{~s}$ at $72^{\circ} \mathrm{C}$, with a melt curve analysis (to verify product identity). From cycle threshold $(\mathrm{Ct})$ values, numbers of CLas genomes were calculated per $\mu \mathrm{g}$ DNA analyzed, based on previously published laboratory analyses (Stover et al., 2015b).

\section{Statistical analyses}

All data including qPCR results (analyzed as $\mathrm{Ct}$ ) were assessed using the nonparametric Kruskal-Wallis test (SAS Institute). Where CLas was found to be nondetectable, a $\mathrm{Ct}$ value of 45 (which is less than one copy of target DNA sequence per sample analyzed) was used in the analyses to provide a reasonable value for calculations. To assess the relationship between growth and CLas titer, regressions were run between the Ct in June 2013 and the percentage trunk cross-sectional area increase over the preceding year (Excel; Microsoft, Redmond, WA). For the inoculativity study, we analyzed data which included all leaves and also using only data from leaves with detectable CLas.

\section{Results and Discussion}

\section{Graft inoculations}

Incidence of both buds on a test tree dying from initial inoculation. Among the budwood test genotypes, rough lemon trees displayed death of both buds from initial inoculations in $33 \%$ of trees vs. $0 \%$ in 'Kuharske' and $10 \%$ in 'Valencia' (Table 1). 'Kuharske' showed no need for rebudding with any source budwood and later showed virtually no mottling or CLas, therefore the main effects of inoculation source genotypes were compared excluding the 'Kuharske' data. As a source genotype the rough lemon budwood had the fewest failures, with only $5 \%$ of trees requiring rebudding vs. $20 \%$ to $35 \%$ for the others (Table 1), although only the citron with $35 \%$ failures was different at $P=0.05$. When assessing individual means for statistically significant interactions, citron and Volkamer lemon source budwood had the highest failure rate in rough lemon test trees, whereas 'Valencia' source budwood had a higher failure than rough lemon or Volkamer lemon for 'Valencia' test trees.

CLas titer in petioles/midribs. At the first budding date, there was remarkable uniformity in CLas titer between the different bud source genotypes and the inoculum provided to each test tree was also virtually identical (data not shown). But at the date of rebudding, the CLas titer was lower (higher $\mathrm{Ct}$ ), and therefore, where rebudding was necessary, both test and source genotypes showed lower mean titers for leaf petioles associated with inoculum buds.

'Kuharske' is a selection of 'Carrizo' which has been previously shown to have few HLB symptoms and develop low pathogen titer (Folimonova et al., 2009), and the 'Kuharske' test trees had very low CLas titer throughout the 15 months of the experiment. However, rough lemon and 'Valencia' had moderate titers by Jan. 2013 which continued through the last assessment and were not statistically different from each other. Therefore, data presented on CLas titer as influenced by main effect of inoculum source excluded 'Kuharske' test trees. Averaged across all trees, with the exception of

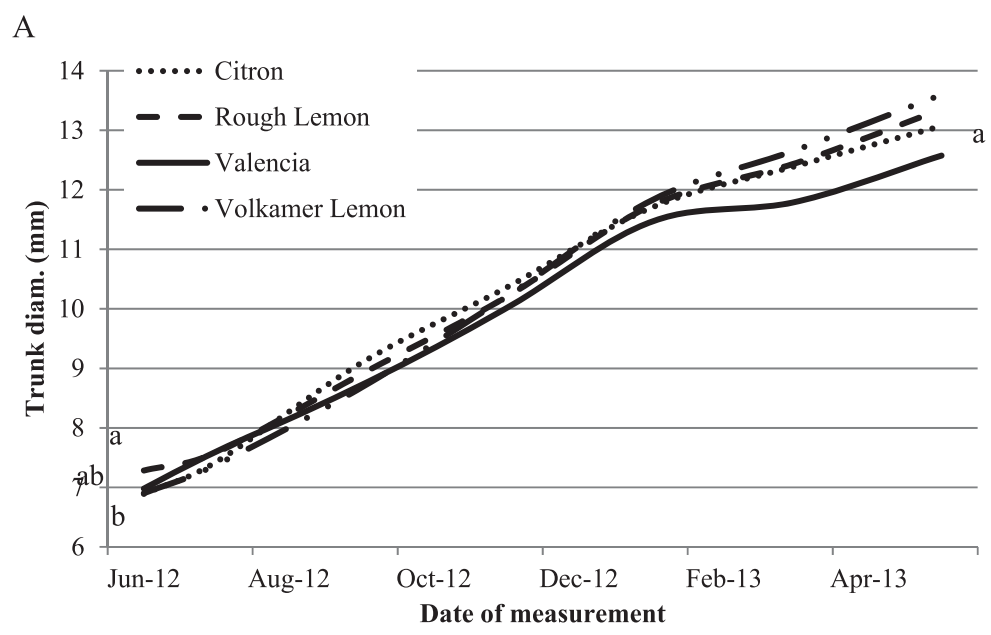

B

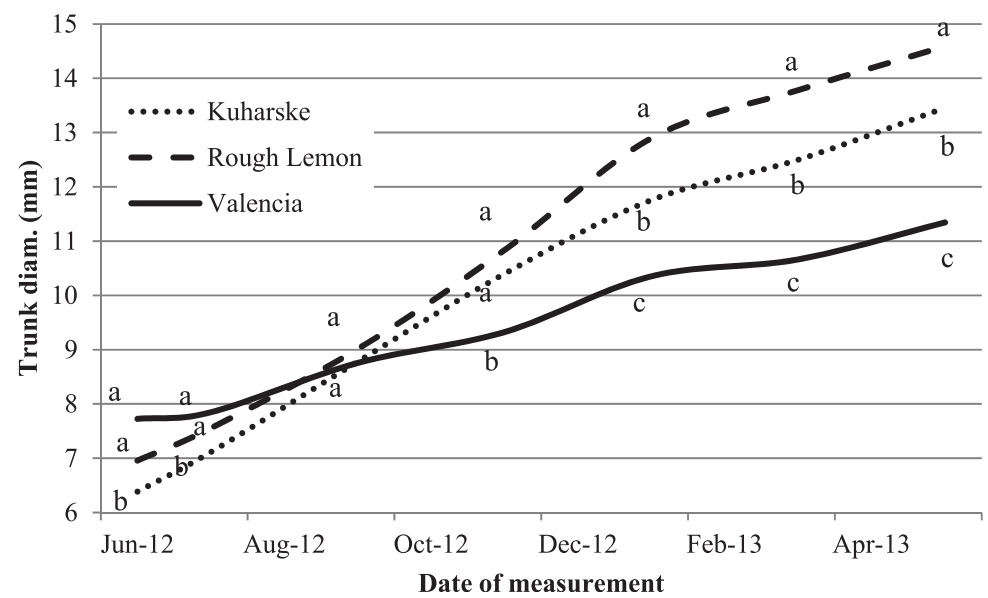

Fig. 1. Trunk diameter averaged across 10 trees each of three citrus genotypes ('Kuharske' citrange, rough lemon, and 'Valencia') double bud inoculated 20 Mar. 2012 with highly huanglongbing symptomatic citron, rough lemon, 'Valencia', or Volkamer lemon. On each date, trunk diameters are not significantly different at the $P=0.05$ level if marked by the same letter. Following May 2012, there was no statistically significant effect of bud inoculum source on growth. There was no significant interaction between inoculated genotype and bud source. (A) Effect of bud inoculum source genotype $(10$ trees $\times 3$ inoculated genotypes $=30$ trees with each budwood source $)$. (B) Effect of test genotype (10 trees $\times 4$ budwood sources $=40$ trees of each genotype). 
'Kuharske', trees inoculated with rough lemon and 'Valencia' buds had significantly higher CLas titers than those inoculated with buds from citron or Volkamer lemon in Jan. and Mar. 2013, but by May 2013 there were no statistical differences in titer as influenced by source genotype.

At 10 months after inoculation (Jan. 2013), the petiolar CLas titers between test trees were statistically different between the resistant 'Kuharske' vs. the susceptible 'Valencia' and tolerant rough lemon, for all inoculum source genotypes except Volkamer lemon. Petiolar CLas titers between test trees were statistically different between 'Kuharske' vs. 'Valencia' and rough lemon for the ensuing 4 months when either 'Valencia' or rough lemon was used as the inoculum source.

There were a few notable interactions between inoculum source and test genotypes. At 10 months after inoculation (Jan. 2013), rough lemon source budwood produced lower CLas titers in 'Kuharske' than did 'Valencia' or citron, although all 'Kuharske' titers were very low. In 'Valencia' test trees inoculated with citron source budwood, titers were never statistically different from 'Valencia' inoculated with 'Valencia' budwood and citron produced higher titer than did rough lemon by May 2013.

Development of distinctive HLB mottle. Negligible mottle was observed before Nov. 2012. 'Kuharske' test trees never displayed more than slight mottle symptoms. Neither citron nor Volkamer lemon source budwood conferred high levels of mottle symptoms to rough lemon or 'Valencia' test genotypes, despite having similar levels of CLas in source budwood. Marked budwood source genotypes by test genotype interactions were observed in 'Valencia' and rough lemon. When assessing test tree by source tree interactions, rough lemon and 'Valencia' source budwood were usually equally effective in inducing CLas titer and HLB blotchy mottle except that rough lemon source budwood produced a greater percentage of HLB mottle than did 'Valencia' budwood in the 'Valencia' test genotype.

Tree growth. In May 2012 (Fig. 1), test trees inoculated with rough lemon were slightly larger in trunk diameter than trees inoculated with citron $(7.3$ vs. $6.9 \mathrm{~mm})$. After that time, main effect of budwood source genotype averaged across test trees was never statistically significant. However, there was a marked difference in tree growth between the test genotypes (Fig. 1). The 'Valencia' trees were significantly larger than the 'Kuharske' trees at the start of the experiment, but grew considerably less than the other test genotypes. Most notably, rough lemon, which displayed greater mottle than 'Valencia' test trees and had similar high CLas titers, increased in trunk diameter much the same as the HLB-resistant 'Kuharske' with very low CLas titer. Calculated from trunk diameter, the trunk cross-sectional area increase from May 2012 to May 2013 was 3.51-fold in 'Kuharske', 3.56-fold in rough lemon, and 1.22 -fold in 'Valencia'. Although rough lemon is known to produce a very vigorous tree, healthy 'Valencia' on rough lemon also grows vigorously. This difference in rough lemon and 'Valencia' growth while displaying similar levels of CLas and HLB symptoms is indicative of the tolerance to HLB displayed by rough lemon (Fan et al., 2012) and some related genotypes (Miles et al., 2016). Regressions were conducted for each test genotype, assessing the relationship between the June $2013 \mathrm{Ct}$ for CLas and the trunk cross-sectional area increase over the preceding year, using only trees with detectable CLas. Much lower $r^{2}$ for rough lemon $\left(r^{2}=0.106, P=0.064\right)$ compared with 'Valencia' $\left(r^{2}=0.383, P<\right.$ 0.0001 ) provides further evidence of some HLB tolerance in rough lemon. The same regression analysis in 'Kuharske' provided an $r^{2}=0.012$ and $P=0.77$.

\section{Detached leaf assays with ACP}

The incidence of CLas-infected ACP and associated titer levels are known to be variable even in colonies raised on highly infected plants (Hall et al., 2016). In our assays, the source ACP populations ranged from $30 \%$ to $80 \%$ infected when using a threshold of $\mathrm{Ct} \leq 36$ (Table 2). For ACP with $\mathrm{Ct} \leq 36$, mean $\mathrm{Ct}$ ranged from 27.9 to 30.8 . At the end of each assay, we did not assay individual ACP from each leaf, thus the inoculum load that each leaf was subjected to is not known. Some mortality of ACP occurred during the 1 -week infestations (Table 2). This is consistent with the results of Ammar et al. (2013), who noted that some mortality of ACP adults occurred in their assays with detached leaves. In our assays, percentage mortality of ACP was significantly higher among those infesting 'Carrizo' as opposed to the four other genotypes during one assay, and was in the highest mortality statistical grouping in all five assays. In a study by Richardson and Hall (2013), ACP maintained on some citranges had shorter life spans, although 'Carrizo' was not among those studied. Regardless of ACP mortality, CLas transmission occurred to at least some leaves of each genotype in each of five different assays with at least $40 \%$ of leaves of each genotype becoming infected in two assays (Table 2). The highest percentages of leaves becoming infected occurred during the June assay-higher transmission rates in this particular assay as opposed to others were probably a consequence of higher

Table 2. Detached leaf assay with ACP. At each date, 10 detached leaves each from 'Flame' grapefruit, 'Temple' tangor, 'Valencia' sweet orange, 'Carrizo' rootstock, and rough lemon rootstock, were subjected to CLas + ACP feeding on detached leaves for 1 week. All ACP were from a colony maintained in the USHRL insectary, with same original CLas source used in the graft inoculation experiments in this report. CLas titer was determined on a sample of source ACP on the date leaves were exposed. The experiment was conducted four times from Mar. 2013 to Feb. 2014, using leaf material from trees with no recent insecticide applications, and verified to cause no ACP mortality following $24 \mathrm{~h}$ of ACP feeding. ACP mortality was assessed at the end of the 1-week experimental feeding period. CLas titer was determined for the petiole/midrib of each leaf, with means across all leaves, and only those with detectable CLas (some amplification of target rDNA).

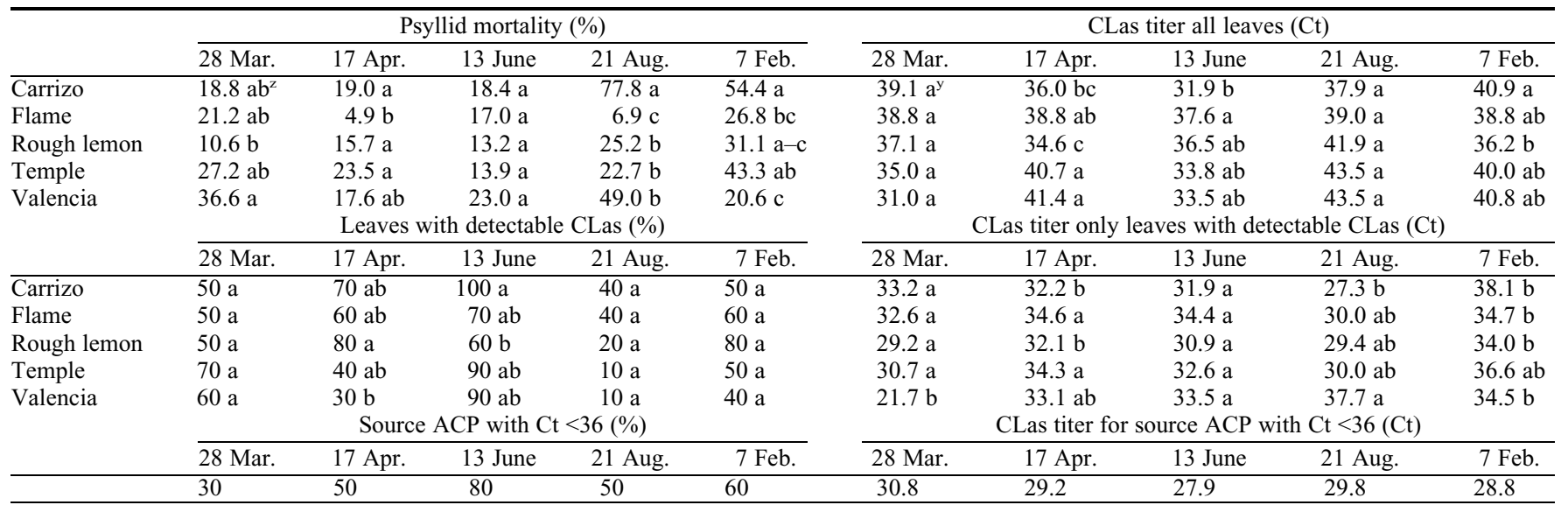

$\mathrm{ACP}=$ Asian citrus psyllid; CLas = Candidatus Liberibacter asiaticus; $\mathrm{Ct}=$ cycle threshold; USHRL $=$ U.S. Horticultural Research Laboratory.

${ }^{\mathrm{z}}$ Within a column and section, means followed by the same letter are not significantly different at $P \leq 0.05$.

${ }^{\mathrm{y}} \mathrm{Ct}$ corresponds to genomes of CLas/ $\mu \mathrm{g}$ DNA as follows: $22=1,800,000,27=76,000,32=3300,37=140$, and $42=6$. 
Table 3. Summary comparing traits of CLas-inoculum budwood source genotypes in an experiment in which three citrus genotypes ('Kuharske' citrange, rough lemon, and 'Valencia') were double bud inoculated with highly HLB symptomatic citron, rough lemon, 'Valencia', or Volkamer lemon. Higher number of "+" symbols for source genotype in each category indicates more favorable characteristics as an inoculum source to impart HLB symptoms and CLas titer.

\begin{tabular}{|c|c|c|c|c|c|c|}
\hline \multirow{2}{*}{$\begin{array}{l}\text { Inoculum } \\
\text { budsource } \\
\text { genotypes }\end{array}$} & \multirow{2}{*}{$\begin{array}{c}\text { CLas titer } \\
\text { in buds used } \\
\text { in inoculation }\end{array}$} & \multirow{2}{*}{$\begin{array}{l}\text { Shoot growth in } \\
\text { infected trees used } \\
\text { as budsource }\end{array}$} & \multirow{2}{*}{$\begin{array}{c}\text { Budtake } \\
\text { without need } \\
\text { for rebudding }\end{array}$} & \multirow{2}{*}{$\begin{array}{l}\text { Production of } \\
\text { mottle symptoms } \\
\text { in recipient plants }\end{array}$} & \multicolumn{2}{|c|}{ Development of CLas titer in recipient plants } \\
\hline & & & & & $\begin{array}{c}\text { At } 10-12 \text { mo. } \\
\text { after inoculation }\end{array}$ & $\begin{array}{l}\text { At } 14 \text { mo. after } \\
\text { inoculation }^{\mathrm{x}}\end{array}$ \\
\hline Citron & +++ & +++ & + & + & + & ++ \\
\hline Rough lemon & +++ & +++ & +++ & +++ & +++ & ++ \\
\hline Valencia & +++ & + & ++ & ++ & +++ & ++ \\
\hline Volkamer lemon & +++ & +++ & ++ & + & + & ++ \\
\hline
\end{tabular}

CLas $=$ Candidatus Liberibacter asiaticus; HLB $=$ huanglongbing.

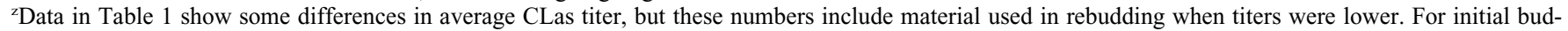
inoculations, all source genotypes had similar titers.

${ }^{\mathrm{y}}$ Data not shown.

${ }^{\mathrm{x}}$ Extracted from data in Table 1.

numbers of CLas infected ACP per leaf in combination with higher titers of CLas per ACP. Over all genotypes and assays, an average of $55 \%$ of leaves developed detectable levels of CLas and the $\mathrm{Ct}$ value associated with infected leaves averaged 32.4 (Table 2). Over all assays, there was relatively little variation among genotypes in the percentage of leaves becoming CLas infected (range from $46 \%$ to $62 \%$ ) or in the titer of CLas developing in infected leaves (Ct range from 31.1 to 32.8). However, there were larger differences in transmission rates among assays with respect to percentages of leaves becoming infected (range $24 \%$ to $82 \%$ ) and average $\mathrm{Ct}$ values of infected leaves (range 29.5 to 35.6). The results generally indicated similar transmission rates of CLas occurred to each of the five genotypes by ACP reared on infected rough lemon. Based on research reports by Hall et al. (2016), detached leaf assays conducted to assess CLas resistance may provide more consistent inoculation if fully expanded flush leaves are used rather than mature leaves, as used here.

\section{Conclusions}

Experiments were conducted to assess factors which might permit more rapid screening for HLB resistance. Consistency of CLas titer and effectiveness in infecting test trees was evaluated using several source genotypes for graft inoculations. Rough lemon and 'Valencia' were much better sources of graft inoculum than citron or Volkamer lemon, even though all four source genotypes had similar CLas titer levels. Rough lemon as source trees for inoculum produced much more budwood than 'Valencia' and had the lowest incidence of bud mortality in test trees, making rough lemon an especially good source of bud inoculum in these studies (Table 3).

Trunk cross-sectional area increase was $3 \times$ higher in 'Kuharske' and rough lemon, compared with 'Valencia': 'Kuharske' had very low levels of CLas (30/ $\mu \mathrm{g}$ DNA),

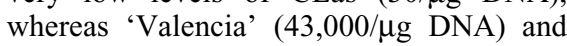
rough lemon $(6700 / \mu \mathrm{g}$ DNA) had relatively high levels. This is indicative of the tolerance to HLB displayed by rough lemon.

In detached leaf assays, where ACP from infected rough lemon were used to inoculate five different genotypes, no major differences were observed among test genotypes in transmission rates of CLas. From a time standpoint, results of the detached leaf assays could be obtained within 2 to 3 weeks as compared with the 11 to 15 months required for our graft inoculations of whole plants. However, this would only be useful in assessing resistance and not tolerance.

\section{Literature Cited}

Albrecht, U. and K.D. Bowman. 2008. Gene expression in Citrus sinensis (L.) Osbeck following infection with the bacterial pathogen Candidatus Liberibacter asiaticus causing huanglongbing in Florida. Plant Sci. 175:291306.

Ammar, E.D., A.J. Walter, and D.G. Hall. 2013. New excised-leaf assay method to test inoculativity of Asian citrus psyllid (Hemiptera: Psyllidae) with Candidatus Liberibacter asiaticus associated with citrus huanglongbing disease. J. Econ. Entomol. 106:25-35.

Bové, J.M. 2006. Huanglongbing: A destructive, newly-emerging, century-old disease of citrus. J. Plant Pathol. 88:7-37.

CDFA (California Dept. of Food and Agr.). 2015. Citrus disease huanglongbing detected in San Gabriel area of Los Angeles County. Apr. 2016. $<$ https://www.cdfa.ca.gov/egov/Press_ Releases/Press_Release.asp?PRnum=15-031.

Fan, J., C. Chen, Q. Yu, A. Khalaf, D.S. Achor, R. H. Brlansky, G.A. Moore, Z.-G. Li, and F.G. Gmitter, Jr. 2012. Comparative transcriptional and anatomical analyses of tolerant rough lemon and susceptible sweet orange in response to 'Candidatus Liberibacter asiaticus' infection. Mol. Plant Microbe Interact. 25:13961407.

Folimonova, S.Y., C.J. Robertson, S.M. Garnsey, S. Gowda, and W.O. Dawson. 2009. Examination of the responses of different genotypes of citrus to huanglongbing (citrus greening) under different conditions. Phytopathology 99:13461354.

Gottwald, T.R. 2010. Current epidemiological understanding of citrus huanglongbing. Annu. Rev. Phytopathol. 48:119-139.

Halbert, S.E., K.L. Manjunath, and M.W. Brodie. 2008. Large-Scale Distribution of Diaphorina citri Kuwayama and citrus huanglongbing in Florida. p. 112-115. (Proc. Int. Res. Conf. Huanglongbing). Apr. 2016. <https://www. plantmanagementnetwork.org/proceedings/ irchlb/2008/presentations/IRCHLB.2.6.pdf>.

Hall, D.G., U. Albrecht, and K.D. Bowman. 2016. Transmission rates of ' $\mathrm{Ca}$. Liberibacter asiaticus' by Asian citrus psyllid are enhanced by the presence and developmental stage of citrus flush. J. Econ. Entomol., doi: 10.1093/jee/tow009.

Miles, G.P., E.W. Stover, C. Ramadugu, M. Keremane, and R.F. Lee. 2016. Apparent tolerance to huanglongbing in Citrus and Citrus-related germplasm. HortScience (in preparation).

Richardson, M.L. and D.G. Hall. 2013. Resistance of Poncirus and Citrus $\times$ Poncirus germplasm to the Asian citrus psyllid. Crop Sci. 53:183188.

Singerman, A. and P. Useche. 2015. Impact of citrus greening on citrus operations in Florida. Apr. 2016. <http://www.crec.ifas.ufl.edu/ extension/economics/pdf/Impact\%20Citrus\% 20Greening\%20web.pdf $>$.

Stover, E., S. Inch, M. Richardson, and D.G. Hall. 2016. Conventional citrus of some scion/rootstock combinations show field tolerance under severe huanglongbing disease pressure. HortScience 51:127-132.

Stover, E. and G. McCollum. 2011. Incidence and severity of huanglongbing and Candidatus Liberibacter asiaticus titer among field-infected citrus cultivars. HortScience 46:1344-1348

Stover, E., T.G. McCollum, R. Driggers, R. Lee, R. Shatters, Jr., Y.P. Duan, M. Ritenour, J.X. Chaparro, and D.G. Hall. 2015a. Resistance and tolerance to Huanglongbing in citrus. Acta Hort. 1065:899-903.

Stover, E., G. McCollum, J. Ramos, and R.G. Shatters, Jr. 2015b. Growth, health and Liberibacter asiaticus titer in diverse citrus scions on mandarin vs. trifoliate hybrid rootstocks in a field planting with severe huanglongbing. Proc. Fla. State Hort. Soc. 127:53-59. 